\title{
Anisotropic Weyl Fermions from Quasiparticle Excitation Spectrum of a 3D Fulde-Ferrell Superfluid
}

\author{
Yong $\mathrm{Xu}^{1}$, Rui-Lin $\mathrm{Chu}^{1}$, and Chuanwei Zhang ${ }^{1 *}$ \\ ${ }^{1}$ Department of Physics, The University of Texas at Dallas, Richardson, Texas 75080, USA
}

\begin{abstract}
Weyl fermions, first proposed for describing massless chiral Dirac fermions in particle physics, have not been observed yet in experiments. Recently, much effort has been devoted to explore Weyl fermions around band touching points of single particle energy dispersions in certain solid state materials (named Weyl semimetals), similar as graphene for Dirac fermions. Here we show that such Weyl semimetals also exist in the quasiparticle excitation spectrum of a three-dimensional (3D) spin-orbit coupled Fulde-Ferrell (FF) superfluid. By varying Zeeman fields, the properties of Weyl fermions, such as their creation and annihilation, number and position, as well as anisotropic linear dispersions around band touching points, can be tuned. We study the manifestation of anisotropic Weyl fermions in sound speeds of FF fermionic superfluids, which are detectable in experiments.
\end{abstract}

PACS numbers: 03.75.Ss, 03.75.Lm, 05.30.Fk

Introduction Weyl fermions [1] are massless chiral Dirac fermions with linear energy dispersions in momentum space. Recently, the existence of Weyl fermions has been explored in various solid state materials, such as Pyrochlore Iridates [2, 3], ferromagnetic compound $\mathrm{HgCr}_{2} \mathrm{Se}_{4}$ [4], multilayer topological insulators 5], photonic crystals [6], as well as in optical lattices [7, 8]. These materials, named as Weyl semimetals, possess band touching points in their single particle energy spectrum, around which the energy dispersions are linear and can be described by chiral Weyl equation. These band touching points (i.e., Weyl nodes) appear in pairs with opposite topological invariance [9]. In contrast to two dimensional (2D) Dirac fermions (e.g., graphene), which are unstable against perturbations that break time-reversal or spatial inversion symmetries, Weyl nodes are stable and the only way to destroy them is to merge two Weyl nodes with opposite topological invariances.

The recent progress in experimental observations of Majorana fermions (half of a regular Dirac fermion) using quasiparticle excitations in solid state topological superconductors [10 13] leads to a nature question: whether Weyl fermions can also be observed in the quasiparticle excitation spectrum (instead of single particle spectrum) of superconductors or superfluids (e.g., ${ }^{3} \mathrm{He} \mathrm{A}$ phase) 9, 14, 15]. While Majorana fermions in superconductors emerge as quasiparticle excitations in real space (inside defects) and low dimensions (1D or 2D), Weyl fermions describe energy dispersions in momentum space in 3D. Therefore the semiconductor/superconductor heterostructures for observing Majorana fermions, where superconductivity is induced through proximity effects, are not suitable for the observation of Weyl fermions.

The recent experimental realization of spin-orbit (SO) coupling [16 21] in ultracold atomic gases provide another platform for exploring a variety of intriguing physics, including topological superfluids with Majorana fermions [22 29]. In particular, the low-temperature phase diagram of spin-orbit coupled Fermi gases is dom- inated by Fulde-Ferrell (FF) superfluids with finite momentum pairing $30-40$ ] in the presence of an in-plane Zeeman field, even in 3D. In $1 \mathrm{D}$ and $2 \mathrm{D}$, such FF superfluids can support Majorana fermions [4 44]. However, whether such FF superfluid state can support Weyl fermion excitations has not been explored.

In this Letter, we show that Weyl fermions can emerge from quasiparticle excitation spectrum of a $3 \mathrm{D}$ FF superfluid. The system we consider is a 3D degenerate Fermi gas with Rashba spin-orbit coupling (in $x y$ plane) and Zeeman fields (in-plane $\left(h_{x}\right)$ and out-of-plane $\left(h_{z}\right)$ ). The in-plane Zeeman field breaks the spatial inversion symmetry of the Fermi surface, yielding finite momentum pairing 32]. The rich phase diagrams in such 3D FF superfluids are obtained. In suitable parameter regions, we find band touching points between particle and hole branches in the quasiparticle excitation spectrum of the FF superfluid, which possess non-zero topological invariances and anisotropic linear dispersions along all three directions, indicating the existence of anisotropic Weyl fermion excitations. The properties of Weyl fermions, including their number and position, creation and annihilation, and anisotropy, can be controlled by varying Zeeman fields and interaction strength between atoms. Finally, we investigate the signature of anisotropic Weyl fermion excitations in the speeds of sound of the FF fermionic superfluids, which are measurable in experiments.

Model and effective Hamiltonian: We consider a 3D Fermi gas with $s$-wave contact interactions. The many-body Hamiltonian can be written as $H=$ $\int d \mathbf{r} \hat{\Psi}^{\dagger}(\mathbf{r}) H_{s}(\hat{\mathbf{p}}) \hat{\Psi}(\mathbf{r})-U \int d \mathbf{r} \hat{\Psi}_{\uparrow}^{\dagger}(\mathbf{r}) \hat{\Psi}_{\downarrow}^{\dagger}(\mathbf{r}) \hat{\Psi}_{\downarrow}(\mathbf{r}) \hat{\Psi}_{\uparrow}(\mathbf{r})$, where the single particle Hamiltonian $H_{s}(\hat{\mathbf{p}})=$ $\frac{\hat{\mathbf{p}}^{2}}{2 m}-\mu+H_{\mathrm{SOC}}(\hat{\mathbf{p}})+H_{z}$ with momentum operator $\hat{\mathbf{p}}=-i \hbar \nabla$, chemical potential $\mu$, attractive interaction strength $U$, and the atom mass $m$; the Rashba SO coupling $H_{\mathrm{SOC}}(\hat{\mathbf{p}})=\alpha(\hat{\mathbf{p}} \times \sigma) \cdot \mathbf{e}_{z}$ with Pauli matrix $\sigma$; the Zeeman field is along $x$ (in-plane) and $z$ (out-of-plane) directions, $H_{z}=h_{x} \sigma_{x}+h_{z} \sigma_{z} . \hat{\Psi}(\mathbf{r})=\left[\hat{\Psi}_{\uparrow}(\mathbf{r}), \hat{\Psi}_{\downarrow}(\mathbf{r})\right]^{T}$ and 


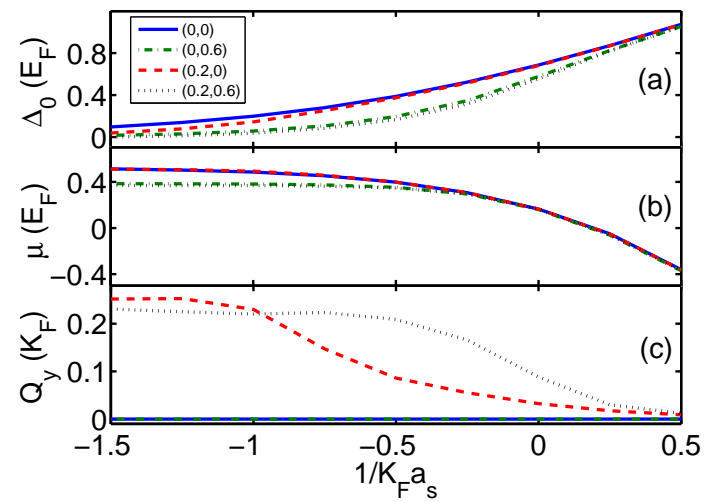

FIG. 1: (Color online) Plot of $\Delta_{0}$ in (a), $\mu$ in (b), and $Q_{y}$ in (c) as a function of $1 / K_{F} a_{s}$ for different parameters $\left(h_{x}, h_{z}\right)$. $\alpha K_{F}=E_{F}$ and the temperature $T=0$.

$\hat{\Psi}_{\nu}^{\dagger}(\mathbf{r})\left(\hat{\Psi}_{\nu}(\mathbf{r})\right)$ is fermionic atom creation (annihilation) operator.

The thermodynamical potential in mean-field approximation can be written as

$$
\begin{aligned}
\Omega= & |\Delta|^{2} / U+\sum_{\mathbf{k}}\left(\hbar^{2}(-\mathbf{k}+\mathbf{Q} / 2)^{2} / 2 m-\mu\right) \\
& -\sum_{\mathbf{k}, \sigma} \frac{1}{2 \beta} \ln \left(1+e^{-\beta E_{\mathbf{k} \sigma}}\right) .
\end{aligned}
$$

Here $E_{\mathbf{k} \sigma}$ is the eigenvalue of $4 \times 4$ Bogoliubov-de Gennes (BdG) Hamiltonian

$$
H_{B}=\left(\begin{array}{cc}
H_{s}(\mathbf{k}+\mathbf{Q} / 2) & \Delta_{0} \\
\Delta_{0} & -\sigma_{y} H_{s}(-\mathbf{k}+\mathbf{Q} / 2)^{*} \sigma_{y}
\end{array}\right),
$$

$\mathbf{Q}=Q_{y} \mathbf{e}_{y}$ is the total momentum of the Cooper pair induced by the deformation of the Fermi surface [32]. The mean-field solutions of $\Delta_{0}, Q_{y}$, and $\mu$ satisfy the saddle point equations $\partial \Omega / \partial \Delta_{0}=0, \partial \Omega / \partial Q_{y}=0$, and the atom number equation $\partial \Omega / \partial \mu=-n$ with a fixed total atom density $n$. To regularize the ultra-violet divergence at large $\mathbf{k}$, we follow the standard procedure [45] $\frac{1}{U}=$ $\frac{m}{4 \pi \hbar^{2} a_{s}}-\int \frac{d \mathbf{k}}{(2 \pi)^{3}} \frac{m}{\hbar^{2} k^{2}}$ with the s-wave scattering length $a_{s}$. The self-consistent solution is obtained through the minimization of the free energy $F=\Omega+\mu n$. The energy unit is chosen as the Fermi energy $E_{F}=\hbar^{2} \mathbf{K}_{F}^{2} / 2 m$ of non-interacting Fermi gases without SO coupling and Zeeman fields with Fermi vector $K_{F}=\left(3 \pi^{2} n\right)^{1 / 3}$.

$B C S-B E C$ crossover and phase diagram: In Fig. 1, we plot the change of $\mu, \Delta_{0}$ and $Q_{y}$ in the BCS-BEC crossover at zero temperature. With increasing $1 / K_{F} a_{s}$, $\Delta_{0}$ increases and $\mu$ decreases, signalling the crossover from BCS superfluids to BEC molecules. In the BEC limit, $\Delta_{0}, \mu$ and $Q_{y}$ become the same for different values of $\left(h_{x}, h_{z}\right)$ since fermion atoms form bound molecules. Henceforth we focus on the BCS region. In this region, $\Delta_{0}$ is smaller when the total Zeeman field $h=\sqrt{h_{x}^{2}+h_{z}^{2}}$ is larger. The nonzero $Q_{y}$ in the presence of $h_{x}$ indicates the existence of Cooper pairings with finite centerof-mass momenta.

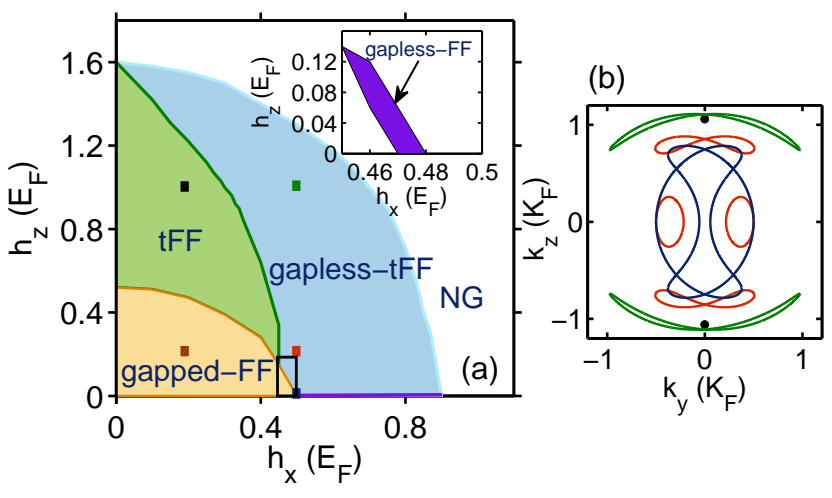

FIG. 2: (a) Mean-field phase diagrams of 3D spin-orbit coupled Fermi gases. The area in the small black box is enlarged in the inset, which shows the gapless FF phase. NG: Normal Gas. (b) Contours of zero energy quasiparticle spectrum in the plane $\left(k_{y}, k_{z}\right)$ with $k_{x}=0$. Here $h_{x}=0.5 E_{F}, h_{z}=0$ (blue line), $h_{z}=0.2 E_{F}$ (red line), $h_{z}=E_{F}$ (green line), and $h_{x}=0.2 E_{F}, h_{z}=E_{F}$ (black points) correspond to blue, red, green, black square points in (a). The brown point in the gapped FF phase has no zero energy excitations. In both figures, $\alpha K_{F}=E_{F}$ and $1 / a_{s} K_{F}=-0.1$.

In Fig. 2(a), we map out the zero-temperature meanfield phase diagram in the $\left(h_{x}, h_{z}\right)$ plane. In 3D Fermi gases, quantum fluctuations generally do not change the phase diagram qualitatively [46, 47]. The uniform superfluid phase (with zero total momentum pairing), which exists at $h_{x}=0$, is replaced by the gapped FF phase [36, 37] in the presence of a small in-plane Zeeman field $h_{x}$ because of the broken inversion symmetry of the Fermi surface. To characterize different phases, we consider the quasiparticle gap $E_{g}$ that represents the energy difference between the minimum of the particle branch and the maximum of the hole branch $\left(E_{g}>0\right.$, gapped; $E_{g} \leq 0$, gapless). The topological FF ( $\left.\mathrm{tFF}\right)$ phase that supports Weyl fermions in the quasiparticle excitation spectrum appears in the large $h_{z}$ region. The topological FF phase is gapped except at Weyl nodes, where $E_{g}=0$. The critical $h_{z}$ for the transition to the tFF phase decreases as $h_{x}$ increases and reaches the minimum $h_{z}=0.14 E_{F}$ at $h_{x}=0.45 E_{F}$. In the absence of $h_{z}$, the gapless FF phase appears with a large range of $h_{x}$ [36, 37], while the region is extremely small (shown in the inset of Fig. 2(a)) in the presence of $h_{z}$. Instead, a new phase called gapless topological FF phase dominates the remaining parameter region because of the large total Zeeman field. This phase possesses non-topological gapless excitations in additional to topological Weyl fermions, which is different from the topological FF phase where Weyl nodes are the only gapless excitations. This phase should also be distinguished from the gapless FF state because its band structure has certain topological property.

Topological FF phase: The transition from gapped FF 

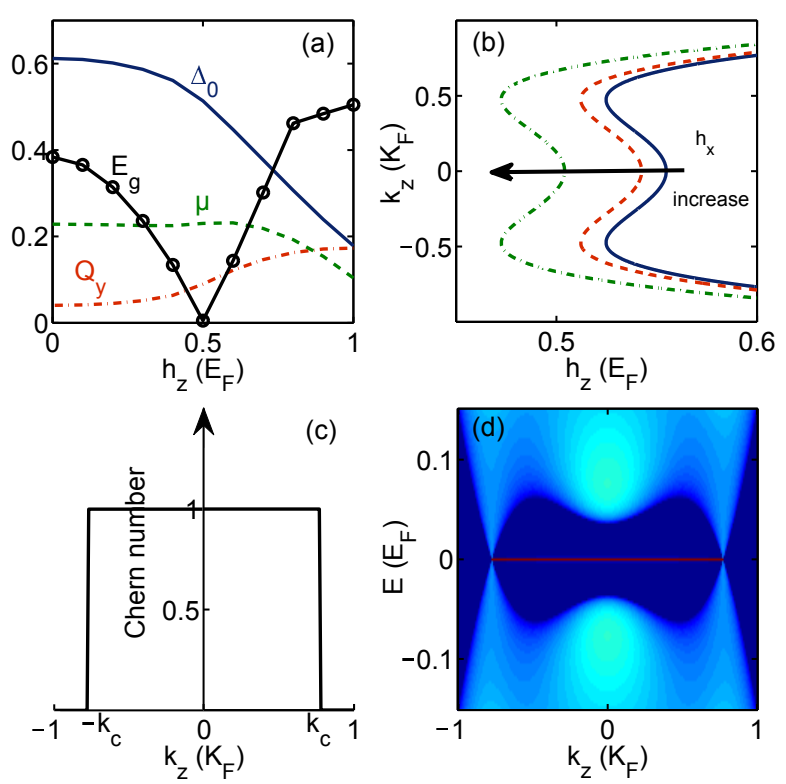

FIG. 3: (Color online) (a) The change of the quasiparticle excitation gap $E_{g}, \Delta_{0}, Q_{y}, \mu$ as a function of $h_{z}$ with $h_{x}=0.2 E_{F}$. (b) Gap closing points $\left(E_{g}=0\right)$ in the $\left(h_{z}, k_{z}\right)$ plane for $h_{x}=0$ (solid blue line), $h_{x}=0.1$ (dashed red line), and $h_{x}=0.2$ (dashed-dot green line). (c) Chern number for a fixed $k_{z}$ plane. (d) displays the density of states, which is calculated via the iterative Green function method [48]. $k_{y}=0$. The light blue region and the red line (Fermi arch) represent bulk and surface excitations, respectively. In (c) and (d), $h_{x}=0.2 E_{F}, h_{z}=0.55 E_{F}$. In all four figures $\alpha K_{F}=E_{F}$, $1 / a_{s} K_{F}=-0.1$.

phase to topological FF phase is characterized by the quasiparticle excitation gap that closes and reopens with increasing $h_{z}$ for a fixed $k_{z}$ and a small $h_{x}$. During this transition, the minimum of the band gap $E_{g}$ occurs at $k_{x}=k_{y}=0$, and the gap closes when

$$
\left(h_{x}+\alpha Q_{y} / 2\right)^{2}+h_{z}^{2}=\left(\hbar^{2} k_{z}^{2} / 2 m-\mu\right)^{2}+\Delta_{0}^{2},
$$

which determines the position $\mathbf{k}_{W}=\left(0,0, k_{c}\right)$ of the Weyl nodes. Such gap close and reopen is shown in Fig. 3(a) for $k_{z}=0$. During this transition, the order parameter $\Delta_{0}$ is still finite even the bulk gap is closed. The finite $Q_{y}$ indicates the FF superfluid. In Fig. 3(b), we plot the Weyl nodes determined by Eq. (3) in the $\left(k_{z}, h_{z}\right)$ plane. For a fixed $h_{z}$ and $h_{x}$, the superfluid is topological when there exists Weyl nodes at certain $k_{z}$. There are two topological regions: $h_{z}>\sqrt{\mu^{2}+\Delta_{0}^{2}-\left(h_{x}+\alpha Q_{y} / 2\right)^{2}}$ with two Weyl nodes and $\sqrt{\Delta_{0}^{2}-\left(h_{x}+\alpha Q_{y} / 2\right)^{2}}<h_{z}<$ $\sqrt{\mu^{2}+\Delta_{0}^{2}-\left(h_{x}+\alpha Q_{y} / 2\right)^{2}}$ with four Weyl nodes. Both critical values for $h_{z}$ decrease with increasing $h_{x}$. To confirm such excitations are Weyl fermions, we examine the energy dispersions around these node points and find they are linear but with different slopes along different directions (see Fig. $4(\mathrm{a})$ ). While the different slopes be-

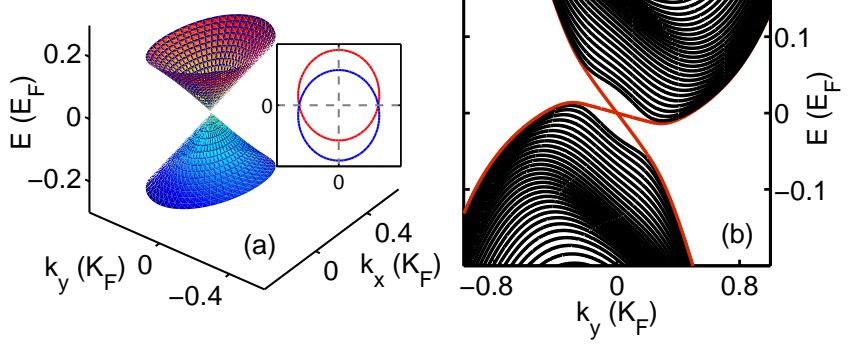

FIG. 4: (Color online) (a) Quasiparticle excitations around the Weyl node $\mathbf{k}_{W}=\left(0,0,0.77 K_{F}\right)$ with $h_{z}=0.55 E_{F}$ and $h_{x}=0.2 E_{F}$. The inset gives the contours of energy with $E=0.1 E_{F}$ ( red line) and $E=-0.1 E_{F}$ (blue line). (b) Quasiparticle excitation spectrum in the gapless topological FF phase as a function of $k_{y}$ with fixed $k_{z}=0.8 K_{F}$ and confinement in the $x$ direction. Black lines are bulk states while the red lines correspond to the surface state. $h_{x}=$ $0.5 E_{F}$ and $h_{z}=0.2 E_{F} . \quad \alpha K_{F}=E_{F}, 1 / a_{s} K_{F}=-0.1$ for both (a) and (b).

tween $k_{z}$ and in-plane directions are due to the $2 \mathrm{D} \mathrm{SO}$ coupling, the difference between $k_{x}$ and $k_{y}$ directions is caused by the finite momentum $Q_{y}$ of the Cooper pairs induced by $h_{x}$. From Eq. (3), we see the properties of Weyl fermions, such as position $\left(k_{c}\right)$, number (2 or 4 ), and their creation and annihilation, can be tuned by varying $h_{z}$ and $h_{x}$. Note that the anisotropy of Weyl fermions can also be tuned by the external magnetic field in solid materials (e.g. Pyrochlore Iridates [3]). In addition, such Weyl fermions appear in pairs with opposite topological charges $N_{c}= \pm 1$ [49].

Weyl nodes can also be regarded as quantum Hall transition points in the momentum space parameterized by $k_{z}$. In the topological FF phase, because the quasiparticle excitations are gapped (except at the Weyl nodes) in the $2 \mathrm{D}$ plane with a fixed $k_{z}$, we can calculate the Chern number for the hole branch for each $k_{z}$ plane

$$
C\left(k_{z}\right)=\frac{1}{2 \pi} \sum_{n} \int d k_{x} d k_{y} \Omega^{n}\left(k_{x}, k_{y}\right)
$$

where $n$ is the index for hole branches, and the Berry curvature [50]

$$
\Omega^{n}=i \sum_{n^{\prime} \neq n}\left[\frac{\left\langle n\left|\partial_{k_{x}} H_{B}\right| n^{\prime}\right\rangle\left\langle n^{\prime}\left|\partial_{k_{y}} H_{B}\right| n\right\rangle-\left(k_{x} \leftrightarrow k_{y}\right)}{\left(E_{n \mathbf{k}}-E_{n^{\prime} \mathbf{k}}\right)^{2}}\right],
$$

and $n^{\prime}$, which is not equal to $n$, runs over the eigenstates of $H_{B}$. For the topological FF phase with two Weyl nodes, we find that $C=1$ when $\left|k_{z}\right|<k_{c}$ and $C=0$ when $\left|k_{z}\right|>k_{c}$ (see Fig. 3 (c)). For the topological FF phase with four Weyl nodes, $C=1$ when $k$ lies between two nodes in the positive or negative $k_{z}$, and $C=0$ otherwise. The Fermi surface lies at zero energy, which is composed of separated Weyl nodes. It is expected that 


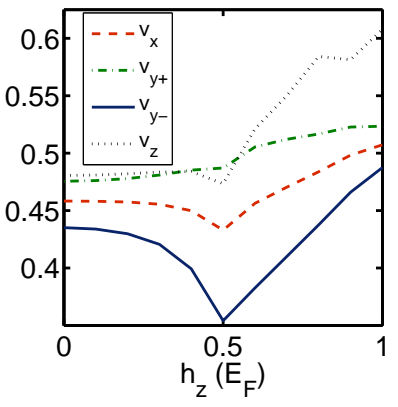

FIG. 5: (Color online) Sound speeds as a function of $h_{z}$. Dashed red and dotted black lines represent the speed along $x$ and $z$ directions. Solid blue and dashed-dot green lines represent sound speeds along negative $y$ and positive $y$ directions, respectively. The unit of sound speed is $v_{F}=\hbar K_{F} / m$. Here $h_{x}=0.2 E_{F}, \alpha K_{F}=E_{F}$, and $1 / a_{s} K_{F}=-0.1$.

there exist chiral edge states [2, 4] in the presence of real space confinement along $x$ or $y$ direction for a fixed $k_{z}$ with nonzero Chern number. For instance, with the confinement along the $x$ direction, there are two chiral edge states whose spectrum intersect at $k_{y}=0$ and $E=0$ ( $k_{z}$ is already taken as a parameter). The zero energy Fermi surface now becomes a line (i.e., Fermi arc) in the $\left(k_{y}, k_{z}\right)$ plane that connects two Weyl nodes (Fig. 3(d)).

Gapless topological FF phase: Topological FF state is gapless by itself, but the gapless points occur only when particle and hole branches touch. With increasing $h_{x}$, the quasiparticle excitation spectrum becomes more asymmetric along the $k_{y}$ direction. Above certain $h_{x}$, certain part of the particle branch of the spectrum may fall below the zero energy, leading to gapless $\mathrm{FF}$ phase with $E_{g}<0$. Note that the band minimum of particle branch and maximum of hole branch do not occur at the same $\mathbf{k}$ in this phase. The gapless FF phase can be either nontopological or topological, depending on whether the particle and hole branches touch at certain $\mathbf{k}_{W}=\left(0,0, k_{c}\right)$ with the linear dispersion. Fig. 2(b) displays the zero energy contours in the $\left(k_{y}, k_{z}\right)$ plane with $k_{x}=0$ for different states. The topological FF phase has separate zero points along the $k_{z}$ axis, while other phases have closed loops. For the gapless FF phase, the closed lines are connected. For the gapless topological FF phase, the particle and hole branch can still touch at certain $\mathbf{k}_{W}$. Instead of a single point, the zero energy contour now cuts close loops in the $\left(k_{x}, k_{y}\right)$ plane and these loops are connected at $\mathbf{k}_{W}$. Away from $\mathbf{k}_{W}$ along the $k_{z}$ direction, particlehole branches do not touch, and the loops in the $\left(k_{x}, k_{y}\right)$ plane are disconnected, which can be clearly seen from

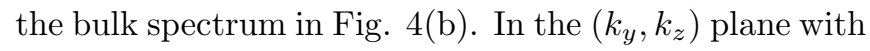
$k_{x}=0$, it forms two connected loop structures around $\mathbf{k}_{W}$, as shown in Fig. 2(b). To confirm the topological nontrivial feature, we calculate the Chern number of the two hole bands and find it is one between two Weyl nodes and zero otherwise. Fig. 4 (b) shows the edge state (red line) in the gapless topological FF phase.

Sound speed of FF superfluids: The anisotropic Weyl fermions in the topological FF phase have two characteristic properties: (i) the anisotropic energy spectrum along all three directions. Furthermore, the spectrum is different even for $\pm k_{y}$ direction, which is special for the FF type of superfluids. (ii) The energy gap closes above a critical $h_{z}$ for the topological phase transition. In experiments, the finite momentum pairing of the Cooper pairs may be measured using noise-correlation imaging [51] or momentum-resolved radio-frequency spectroscopy [35]. Here we propose the above two characteristic properties of Weyl fermions should manifest in the sound speeds of the underlying FF fermionic superfluids, which can be probed by observing the propagation of a localized density perturbation created by a laser beam, as demonstrated in previous experiments [52, 53]. The sound speed can be obtained by calculating Gaussian fluctuations around the saddle point $\left(\Delta_{0}, Q_{y}\right)$ of the thermodynamical potential. Specifically, the speed of sound along $\eta$ direction is defined as $v_{\eta}=\left|\lim _{q \rightarrow 0} \partial \omega(q) / \partial q_{\eta}\right|$, where $\omega(q)$ is bosonic gapless collective excitation spectrum [54] (see supplementary information). Around the Weyl nodes, where the quasiparticle gap closes, we expect a sharp change of the sound speed because of the strong fluctuations around the phase transition points. In Fig. [5 we see the speeds of sound $v_{i}$ are anisotropic along all three different directions, indicating anisotropic quasiparticle spectrum. More interestingly, the sound speeds along the positive and negative $y$ directions are also different, indicating asymmetric spectrum along the $y$ direction due to the finite momentum Cooper pairing. $v_{i}$ has the minimum at the topological phase transition boundary (see Fig. 2(a)) to the topological FF phase with Weyl fermions. Therefore, we conclude that such anisotropic speeds of sound and the minimum located at the phase transition boundary provide strong evidence of Weyl fermions in a FF superfluid. In experiments, we consider a typical parameter with ${ }^{40} \mathrm{~K}$ atoms and density $n=5 \times 10^{12} \mathrm{~cm}^{-3}$, yielding the Fermi energy $E_{F}=h \times 3.5$ $\mathrm{KHz}$ and Fermi velocity $v_{F}=8.3 \mathrm{~mm} / \mathrm{s}$. The $\mathrm{SO}$ coupling and Zeeman field can be created by Raman coupling between atomic hyperfine states. The strength of the Zeeman field may be tuned by the detuning and intensity of Raman lasers [16 21, 55].

Acknowledgement: We would like to thank Xuele Liu, Li Mao, Lin Dong, Ying Hu for helpful discussion and critical comments. This work is supported by ARO (W911NF-12-1-0334), AFOSR (FA9550-13-1-0045), and NSF-PHY (1249293).

\footnotetext{
* Corresponding Author, Email: chuanwei.zhang@utdallas.edu
} 
[1] H. Weyl, Zeitschrift für Physik 56, 330 (1929).

[2] X. Wan, A. M. Turner, A. Vishwanath, and S. Y. Savrasov, Phys. Rev. B 83, 205101 (2011).

[3] V. Aji, Phys. Rev. B 85, 241101(R) (2012).

[4] G. Xu, H. Weng, Z. Wang, X. Dai, and Z. Fang, Phys. Rev. Lett. 107, 186806 (2011).

[5] A. A. Burkov and L. Balents, Phys. Rev. Lett. 107,127205 (2011).

[6] L. Lu, L. Fu, J. D. Joannopoulos, and M. Soljačić, Nature photonics 7, 294 (2013).

[7] D. Bercioux, D. F. Urban, H. Grabert, and W. Häusler, Phys. Rev. A 80, 063603 (2009).

[8] Z. Lan, N. Goldman, and P. Öhberg, Phys. Rev. B 85, 155451 (2012).

[9] G. E. Volovik, The Universe in a Helium Droplet (Clarendon Press, Oxford, 2003).

[10] V. Mourik, K. Zuo, S. M. Frolov, S. R. Plissard, E. P. A. M. Bakkers, L. P. Kouwenhoven, Science 336, 1003 (2012).

[11] M. T. Deng, C. L. Yu, G. Y. Huang, M. Larsson, P. Caroff, and H. Q. Xu, Nano Lett. 12, 6414 (2012).

[12] A. Das, Y. Ronen, Y. Most, Y. Oreg, M. Heiblum, and H. Shtrikman, Nat. Phys. 8, 887 (2012).

[13] L. P. Rokhinson, X. Liu, and J. K. Furdyna, Nat. Phys. 8, 795 (2012).

[14] M. Gong, S. Tewari, and C. Zhang, Phys. Rev. Lett. 107, 195303 (2011).

[15] K. Seo, C. Zhang, and S. Tewari, Phys. Rev. A 87, 063618 (2013).

[16] Y. -J. Lin, K. Jiménez-García, and I. B. Spielman, Nature (London) 471, 83 (2011).

[17] P. Wang, Z. -Q. Yu, Z. Fu, J. Miao, L. Huang, S. Chai, H. Zhai, and J. Zhang, Phys. Rev. Lett. 109, 095301 (2012).

[18] L. W. Cheuk, A. T. Sommer, Z. Hadzibabic, T. Yefsah, W. S. Bakr, and M. W. Zwierlein, Phys. Rev. Lett. 109, 095302 (2012).

[19] J. -Y. Zhang, S. -C. Ji, Z. Chen, L. Zhang, Z. -D. Du, B. Yan, G. -S. Pan, B. Zhao, Y. -J. Deng, H. Zhai, S. Chen, and J. -W. Pan, Phys. Rev. Lett. 109, 115301 (2012).

[20] C. Qu, C. Hamner, M. Gong, C. Zhang, and P. Engels, Phys. Rev. A 88, 021604(R) (2013).

[21] R. A. Williams, M. C. Beeler, L. J. LeBlanc, K. JiménezGarcía, and I. B. Spielman, Phys. Rev. Lett. 111, 095301 (2013).

[22] C. Zhang, S. Tewari, R. M. Lutchyn, and S. Das Sarma, Phys. Rev. Lett. 101, 160401 (2008).

[23] M. Sato, Y. Takahashi, and S. Fujimoto, Phys. Rev. Lett. 103, 020401 (2009).

[24] S. -L. Zhu, L. -B. Shao, Z. D. Wang, and L. -M. Duan, Phys. Rev. Lett. 106, 100404 (2011).

[25] L. Jiang, T. Kitagawa, J. Alicea, A. R. Akhmerov, D. Pekker, G. Refael, J. I. Cirac, E. Demler, M. D. Lukin, and P. Zoller, Phys. Rev. Lett. 106, 220402 (2011).

[26] X. -J. Liu, and H. Hu, Phys. Rev. A 85, 033622 (2012).

[27] K. Seo, L. Han, and C. A. R. Sá de Melo, Phys. Rev.
Lett. 109, 105303 (2012).

[28] M. Gong, G. Chen, S. Jia, and C. Zhang, Phys. Rev. Lett. 109, 105302 (2012).

[29] X. -J. Liu, K. T. Law, and T. K. Ng, arXiv:1304.0291.

[30] P. Fulde, and R. A. Ferrell, Phys. Rev. 135, A550 (1964).

[31] A. I. Larkin, and Yu. N. Ovchinnikov, Zh. Eksp. Teor. Fiz. 47, 1136 (1964) [Sov. Phys. JETP 20, 762 (1965)].

[32] Z. Zheng, M. Gong, X. Zou, C. Zhang, and G. Guo, Phys. Rev. A 87, 031602(R) (2013).

[33] F. Wu, G.-C. Guo, W. Zhang, and W. Yi, Phys. Rev. Lett. 110, 110401 (2013).

[34] X.-J. Liu and H. Hu, Phys. Rev. A 87, 051608(R) (2013).

[35] Z. Fu, L. Huang, Z. Meng, P. Wang, X.-J. Liu, H. Pu, H. Hu, and J. Zhang,, Phys. Rev. A 87, 053619 (2013).

[36] L. Dong, L. Jiang, and H. Pu, New Journal of Physics 15, 075014 (2013).

[37] H. Hui and X.-J. Liu, New Journal of Physics 15, 093037 (2013).

[38] M. Iskin, Phys. Rev. A 88, 013631 (2013).

[39] Y. Xu, C. Qu, M. Gong, and C. Zhang, Phys. Rev. A

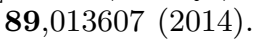

[40] X. -J. Liu, Phys. Rev. A 88, 043607 (2013).

[41] C. Qu, Z. Zheng, M. Gong, Y. Xu, L. Mao, X. Zou, G. Guo, and C. Zhang, Nature Communications 4, 2710 (2013).

[42] W. Zhang and W. Yi, Nature Communications 4, 2711 (2013).

[43] X. -J. Liu and H. Hu, Phys. Rev. A 88, 023622 (2013).

[44] C. Chen, Phys. Rev. Lett. 111, 235302 (2013).

[45] S. Giorgini, L. P. Pitaevskii, S. Stringari, Rev. Mod. Phys. 80, 1215 (2008).

[46] L. He, X.-G. Huang, H. Hu, and X.-J. Liu, Phys. Rev. A 87, $053616(2013)$.

[47] J. P. A. Devreese and J. Tempere, arXiv:1310.3840v1.

[48] X. Dai, T. L. Hughes, X.-L. Qi, Z. Fang, and S.-C. Zhang, Phys. Rev. B 77, 125319 (2008).

[49] The topological charge is defined as $N_{c}=$ $\frac{1}{24 \pi^{2}} e_{\mu \nu \lambda \gamma} \operatorname{tr} \int_{\sum_{a}} d S^{\gamma} \mathscr{G} \partial_{p_{\mu}} \mathscr{G}^{-1} \mathscr{G} \partial_{p_{\nu}} \mathscr{G}^{-1} \mathscr{G} \partial_{p_{\lambda}} \mathscr{G}^{-1}$ [9], where the Green function $\mathscr{G}^{-1}=i k_{0}-H_{B}(\mathbf{k})$, the integral is over the $3 \mathrm{D}$ surface $\sum_{c}$ embracing the isolated Weyl node $\mathbf{k}_{W}=\left(0,0, k_{c}\right)$, and tr stands for the trace in the spin space.

[50] D. Xiao, M.-C. Chang, and Q. Niu, Rev. Mod. Phys. 82, 1959 (2010).

[51] M. Greiner, C. A. Regal, J. T. Stewart, and D. S. Jin, Phys. Rev. Lett. 94, 110401 (2005).

[52] M. R. Andrews, D. M. Kurn, H.-J. Miesner, D. S. Durfee, C. G. Townsend, S. Inouye, and W. Ketterle, Phys. Rev. Lett. 79, 553 (1997).

[53] J. Joseph, B. Clancy, L. Luo, J. Kinast, A. Turlapov, and J. E. Thomas, Phys. Rev. Lett. 98, 170401 (2007).

[54] J. R. Engelbrecht, M. Randeria, and C. A. R. Sáde Melo, Phys. Rev. B 55, 15153 (1997).

[55] C. Zhang, Phys. Rev. A 82, 021607(R) (2010).

\section{SUPPLEMENTARY MATERIALS}

In the main text we propose to detect the topological FF state by measuring the anisotropic sound speeds, which show the signatures of finite momentum pairings as well as topological phase transition. Here we present the calculation details. 
In quantum field theory, the partition function can be written as $Z=\operatorname{Tr}\left(e^{-\beta H}\right)=\int D(\bar{\psi}, \psi) e^{-S_{\text {eff }}[\bar{\psi}, \psi]}$ with $\beta=1 / k_{B} T$. The effective action is

$$
S_{e f f}[\bar{\psi}, \psi]=\int_{0}^{\beta} d \tau\left(\int d \mathbf{r} \sum_{\sigma} \bar{\psi}_{\sigma}(\mathbf{r}, \tau) \partial_{\tau} \psi_{\sigma}(\mathbf{r}, \tau)+H(\bar{\psi}, \psi)\right),
$$

where $\int d \tau$ is an integral over the imaginary time $\tau$ and $H(\bar{\psi}, \psi)$ is obtained by replacing $\hat{\Psi}_{\sigma}^{\dagger}$ and $\hat{\Psi}_{\sigma}$ with Grassman field number $\bar{\psi}_{\sigma}$ and $\psi_{\sigma}$. We can transform the quartic interaction term to quadratic one by Hubbard-Stratonovich transformation, where the order parameter $\Delta(\mathbf{r}, \tau)$ is defined. By integrating out fermion fields, the partition function becomes $Z=\int D(\bar{\Delta}, \Delta) e^{-S_{e f f}[\bar{\Delta}, \Delta]}$, where the effective action can be written as

$$
S_{e f f}[\bar{\Delta}, \Delta]=\int_{0}^{\beta} d \tau \int d \mathbf{r}\left(\frac{|\Delta|^{2}}{U}\right)-\frac{1}{2} \ln \operatorname{det} G^{-1} .
$$

Here the inverse single particle Green function $G^{-1}=-\partial_{\tau}-H_{B}$ in the Nambu-Gor'kov representation with $4 \times 4$ Bogoliubov-de Gennes (BdG) Hamiltonian

$$
H_{B}=\left(\begin{array}{cc}
H_{s}(\hat{\mathbf{p}}) & \Delta(\mathbf{r}, \tau) \\
\Delta(\mathbf{r}, \tau) & -\sigma_{y} H_{s}(\hat{\mathbf{p}})^{*} \sigma_{y}
\end{array}\right) .
$$

Assume that a mean-field solution has the $\mathrm{FF}$ form $\Delta(\mathbf{r}, \tau)_{0}=e^{i Q_{y} y} \Delta_{0}$ with the space independent $\Delta_{0}$ due to the in-plane Zeeman field that deforms the Fermi surface along the $y$ direction, leading to finite momentum pairings along that direction [1]. This form of $\Delta(\mathbf{r}, \tau)$ yields the thermodynamical potential in the main text by Fourier transformation and summing the Matsubara frequency.

To determine the collective modes, we explore Gaussian fluctuations around the saddle point $\Delta(\mathbf{r}, \tau)_{0}$ by separating the pairing field as $\Delta(\mathbf{r})=\Delta(\mathbf{r}, \tau)_{0}+A(\mathbf{r}, \tau)$ with fluctuation field $A$. Expanding $S_{\text {eff }}$ to the second order, we obtain $S_{\text {eff }}=S_{\text {eff }}\left[\Delta_{0}\right]+\frac{1}{2} \sum_{q} \eta^{\dagger} M \eta$. Here $\eta^{\dagger}=\left(A^{*}(q) A(-q)\right)$ with Fourier transformation $A(q)$ of $A(\mathbf{r}, \tau) . q=\left(\mathbf{q}, i q_{m}\right)$ with $q_{m}=i 2 m \pi / \beta$. The $2 \times 2$ inverse fluctuation propagator reads

$$
\begin{aligned}
M_{11}(q) & =M_{22}(-q) \\
& =\frac{1}{U}+\frac{1}{2} \sum_{k} \operatorname{tr}\left[\mathcal{G}_{0}(q+k)_{11} \mathcal{G}_{0}(k)_{22}\right], \\
M_{12}(q) & =\frac{1}{2} \sum_{k} \operatorname{tr}\left[\mathcal{G}_{0}(q+k)_{12} \mathcal{G}_{0}(k)_{12}\right] \\
M_{21}(q) & =\frac{1}{2} \sum_{k} \operatorname{tr}\left[\mathcal{G}_{0}(q+k)_{21} \mathcal{G}_{0}(k)_{21}\right]
\end{aligned}
$$

where $\mathcal{G}_{0}(k)$ is the Fourier transformation of $G(\mathbf{r}, \tau) ; \mathcal{G}_{0}(k)_{i j}$ indicates the $2 \times 2$ block matrix of $\mathcal{G}_{0}(k)$. $\sum_{k}$ represents summation over Matsubara frequency and momentum $\mathbf{k}$. It is important to note that since the analytical expression of $\mathcal{G}_{0}(k)$ cannot be obtained, we calculate the summation over Matsubara frequency numerically.

The dispersion $\omega(\mathbf{q})$ can be $\operatorname{determined}$ by $\operatorname{det}[M[\omega(\mathbf{q}), \mathbf{q}]]=0$ where $i \omega_{n}$ has been taken analytically to $\omega(\mathbf{q})+i 0^{+}$ at zero temperatures. To decouple the amplitude and phase fluctuations, we define $A(x)=\rho(x)+i \delta \theta(x)$, where $\rho(x)$ and $\delta \theta(x)$ are all real. Now the second order effective action is

$$
S_{\text {eff }}^{(2)}=\frac{1}{2} \sum_{q}(\rho(-q) \delta \theta(-q)) N(q)\left(\begin{array}{c}
\rho(q) \\
\delta \theta(q)
\end{array}\right)=\frac{1}{2} \sum_{q}(\rho(-q) \delta \theta(-q))\left(\begin{array}{cc}
N_{11} & N_{12} \\
N_{21} & N_{22}
\end{array}\right)\left(\begin{array}{c}
\rho(q) \\
\delta \theta(q)
\end{array}\right),
$$

where

$$
\begin{aligned}
& N_{11}=M_{11}(q)+M_{22}(q)+M_{12}(q)+M_{21}(q), \\
& N_{12}=i M_{11}(q)-i M_{12}(q)+i M_{21}(q)-i M_{22}(q), \\
& N_{21}=-i M_{11}(q)-i M_{12}(q)+i M_{21}(q)+i M_{22}(q), \\
& N_{22}=M_{11}(q)+M_{22}(q)-M_{12}(q)-M_{21}(q) .
\end{aligned}
$$


To study gapless low energy excitations, we make a small q and $\omega$ expansion [2] using

$$
\begin{aligned}
& N_{11}=A^{11}+\sum_{\sigma=x, y, z} q_{\sigma}^{2} B_{\sigma}^{11}-\omega^{2} C^{11}+\ldots, \\
& N_{22}=\sum_{\sigma=x, y, z} q_{\sigma}^{2} B_{\sigma}^{22}-\omega^{2} C^{22}+\ldots \\
& N_{12}=N_{21}^{*}=-i \omega C^{12}+i q_{y} D_{y}^{12}+\ldots
\end{aligned}
$$

Here $D_{y}^{12}$ comes from the nonzero momenta of Cooper pairs along the $y \operatorname{direction}$. The equation $\operatorname{det}(N)=N_{11} N_{22}-$ $\left|N_{12}\right|^{2}=0$ leads to

$$
\left[A^{11} C^{22}+\left(C^{12}\right)^{2}\right] \omega^{2}-2 \omega q_{y} C^{12} D_{y}^{12}+q_{y}^{2}\left(D_{y}^{12}\right)^{2}-A^{11} \sum_{\sigma} q_{\sigma}^{2} B_{\sigma}^{22}=0
$$

For a special example of a uniform superfluid phase, $D_{y}^{12}=0, B_{x}^{22}=B_{y}^{22}$, and the collective mode dispersion is

$$
\omega(\mathbf{q})=\sqrt{\frac{A^{11}\left[B_{x}^{22}\left(q_{x}^{2}+q_{y}^{2}\right)+B_{z}^{22} q_{z}^{2}\right]}{A^{11} C^{22}+\left(C^{12}\right)^{2}}} .
$$

The sound speed along the $\sigma$ direction with $\sigma=x, y, z$ is

$$
s_{\sigma}=\sqrt{\frac{A^{11} B_{\sigma}^{22}}{A^{11} C^{22}+\left(C^{12}\right)^{2}}},
$$

which is anisotropic between $z$ and in-plane directions because of the 2D SO coupling. In the absence of spin-orbit coupling, $B_{x}^{22}=B_{y}^{22}=B_{z}^{22}$ and the formula reduces to the well-known result [2]. For a general case of a FF superfluid, $D_{y}^{12} \neq 0$, the dispersion along different directions are

$$
\begin{aligned}
\omega\left(q_{x}, q_{y}=0, q_{z}=0\right) & =v_{x}\left|q_{x}\right|, \\
\omega\left(q_{x}=0, q_{y}=0, q_{z}\right) & =v_{z}\left|q_{z}\right|, \\
\omega\left(q_{x}=0, q_{y}>0, q_{z}=0\right) & =v_{y+} q_{y}, \\
\omega\left(q_{x}=0, q_{y}<0, q_{z}=0\right) & =-v_{y} q_{y},
\end{aligned}
$$

where the speeds of sound are

$$
\begin{aligned}
v_{\mu} & =\sqrt{\frac{A^{11} B_{\mu}^{22}}{A^{11} C^{22}+\left(C^{12}\right)^{2}}}, \\
v_{y+} & =\left(-E+\sqrt{E^{2}+4 F}\right) / 2, \\
v_{y-} & =\left(E+\sqrt{E^{2}+4 F}\right) / 2,
\end{aligned}
$$

with $\mu=x, z$, and

$$
\begin{aligned}
& E=-\frac{2 C^{12} D_{y}^{12}}{A^{11} C^{22}+\left(C^{12}\right)^{2}}, \\
& F=-\frac{\left(D_{y}^{12}\right)^{2}-A^{11} B_{y}^{22}}{A^{11} C^{22}+\left(C^{12}\right)^{2}} .
\end{aligned}
$$

We see that the difference of the sound speeds between $x$ and $y$ directions originates from $D_{y}^{12}$ due to finite momentum pairings, which also leads to the speed's difference, equal to $E$ proportional to $D_{y}^{12}$, along the positive and negative $y$ directions.

* Corresponding Author, Email: chuanwei.zhang@utdallas.edu

[1] Y. Xu, C. Qu, M. Gong, and C. Zhang, Phys. Rev. A 89,013607 (2014).

[2] J. R. Engelbrecht, M. Randeria, and C. A. R. Sáde Melo, Phys. Rev. B 55, 15153 (1997). 